\title{
Hernia de Amyand: presentación de un caso
}

\author{
Juan David Molina-Marín', Erika Tatiana Jaramillo-Rojas², Giovanny Vergara-Osorioº Juan Carlos Marín-Marmolejo ${ }^{4}$
}

\section{RESUMEN}

La hernia inguinal es la protrusión de un órgano o fascia a través de la pared de la cavidad que lo contiene y es una de las situaciones más frecuentes que debe enfrentar el cirujano. Por lo general, el contenido de las hernias es epiplón o intestino delgado, pero ocasionalmente se puede observar el apéndice cecal, condición que se denomina "Hernia de Amyand". Esta es una situación infrecuente y de difícil diagnóstico, que generalmente se hace durante la intervención quirúrgica. Se presenta el caso de una hernia de Amyand en un hombre de 40 años que consultó por un cuadro de 10 horas de dolor en la región inguinal derecha, con masa palpable, dolorosa e irreductible; en la cirugía se encontró una hernia que contenía epiplón y el apéndice cecal con cambios inflamatorios. Se hicieron apendicectomía y hernioplastia inguinal por vía preperitoneal con aplicación de malla. No hubo complicaciones intraoperatorias y la evolución fue satisfactoria.

\section{PALABRAS CLAVE}

Hernia Inguinal; Hernia de Amyand

\section{SUMMARY}

\section{Amyand hernia. Case report}

Inguinal hernia is the protrusion of an organ or fascia through the wall of the cavity that contains it. It is one of the most frequent situations that surgeons must face. Typically, the content of herniary sac is omentum or small bowel, but occasionally it includes the cecal

1 Estudiante de Medicina. Coordinador del Semillero de Cirugía e Investigación (SECIN), Facultad de Ciencias para la Salud, Universidad de Caldas, Manizales, Colombia.

2 Residente de Cirugía general, Universidad de Caldas, Manizales, Colombia.

3 Cirujano general, Universidad de Caldas, Manizales, Colombia.

4 Cirujano de Colon y Recto. Coordinador docente del Semillero de Cirugía e Investigación (SECIN). Docente, Universidad de Caldas, Manizales, Colombia Correspondencia: Juan David Molina-Marín; juan.521213064@ucaldas.edu.co

Recibido: septiembre 13 de 2016

Aceptado: octubre 15 de 2016

Cómo citar: Molina-Marín JD, Vergara-Osorio G, Marín-Marmolejo JC, Jaramillo-Rojas ET. Hernia de Amyand: presentación de un caso. latreia. 2017 0ct-Dic;30(4):443-447. D0I 10.17533/udea.iatreia.v30n4a08. 
appendix, a condition called "Amyand hernia". Diagnosis is usually established during surgical intervention. We present the case of an Amyand hernia in a 40 year-old male, who consulted by pain in the right inguinal region, lasting 10 hours. On physical examination a palpable, painful and irreducible mass was found. During surgery, the sac was observed to contain omentum and the cecal appendix, the latter with inflammatory changes. Pre-peritoneal inguinal hernia repair with application of a mesh was performed. No intra-operatory complications occurred and the outcome was satisfactory.

\section{KEY WORDS}

Amyand Hernia; Inguinal Hernia

\section{RESUMO}

\section{Hérnia de Amyand. apresentação de um caso}

A hérnia inguinal é a protrusão de um órgão ou fáscia através da parede da cavidade que os contém e é um dos procedimentos cirúrogicos mais frequentes aos que vai enfrentar o cirurgião. Comumente o conteúdo das hérnias é Epiplon ou intestino delgado e em poucas ocasiões se pode observar a apêndice cecal, condição que se denomina "Hérnia de Amyand". Esta é uma situação pouco frequente e de difícil diagnóstico, que geralmente se diagnostica durante a intervenção cirúrgica. Se apresentou o caso de uma hérnia de Amyand em um paciente masculino de 40 anos de idade que consultou por quadro de 10 horas de dor na região inguinal direita, com massa palpável, dolorosa e irredutível; foi levado a cirurogia e foi durante a qual se encontrou uma hérnia com conteúdo de Epiplon e apênđice cecal com câmbios inflamatórios, se realizou apendicectomia e hernioplastia inguinal por via pré-peritoneal com aplicação de malha. Não se apresentaram complicações intraoperatórias e a evolução foi satisfatória.

\section{PALAVRAS CHAVE}

Ciruroia; Diagnóstico; Hérnia Inguinal; Hérnia de Amyand

\section{INTRODUCCIÓN}

Se conoce como hernia de Amyand el hallazgo del apéndice cecal en el interior del saco herniario; lo más frecuente es que esté normal, pero también puede estar inflamado, gangrenado o perforado $(1,2)$.

En 1731 Garangeot fue el primero en encontrar el apéndice cecal no inflamado dentro del saco peritoneal de una hernia inguinal indirecta, pero la denominación "Hernia de Amyand" se debe al cirujano inglés Claudius Amyand (1681-1740) quien el 6 de diciembre de 1735 intervino un niño de 11 años por una hernia inguinal encarcelada que contenía el apéndice cecal perforado; dicho caso fue registrado como el primero de este tipo (3-5).

Del total de hernioplastias en adultos, la frecuencia de aparición del apéndice normal dentro del saco de la hernia inguinal es del 1 \% y la del apéndice inflamado, del 0,1 \% (6-8). En ocasiones puede estar asociada a pródromos con dolor en el hemiabdomen inferior. Entre los diagnósticos diferenciales se deben incluir el infarto epiploico, la hernia de Ritcher, la adenitis inguinal y la epididimitis. Como el cuadro clínico es inespecífico y es una enfermedad infrecuente, el diagnóstico por lo general es intraoperatorio $(3,9,10)$. Con la presentación de este caso informamos nuestra experiencia en el tratamiento de dicha hernia, con apendicectomía y utilización de malla protésica, lo que es muy debatido en la literatura.

\section{PRESENTACIÓN DEL CASO}

Hombre de 40 años que consultó por cuadro de 10 horas de dolor en la región inguinal derecha, con masa palpable dolorosa e irreductible, sin signos de obstrucción intestinal; tenía leucocitosis (13 100/ $\mu \mathrm{L})$, neutrofilia $(79 \%$ y proteína C reactiva elevada. En la intervención se halló una hernia con contenido de epiplón y apéndice cecal con cambios inflamatorios mínimos (edematosa) en su porción distal (figura1); se hicieron apendicectomía y hernioplastia inguinal por vía preperitoneal con la técnica de Nyhus con aplicación de malla de polipropileno de baja densidad de $15 \times 15 \mathrm{~cm}$, sin complicaciones operatorias; fue dado de alta al día siguiente y controlado una semana después, sin hallar complicaciones postoperatorias. 


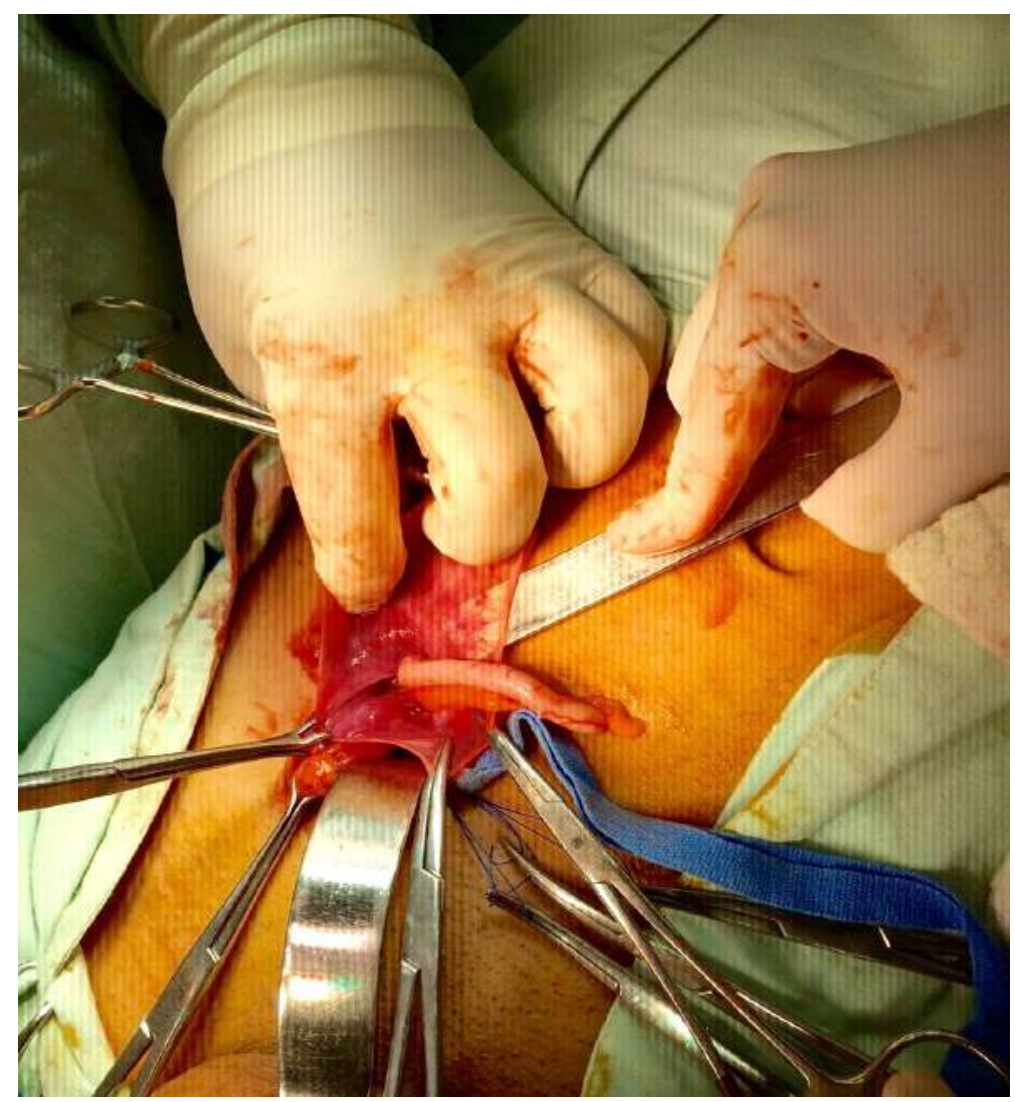

\section{Figura 1. Saco herniario indirecto abierto, apéndice cecal en su interior, con cambios inflamatorios distales (foto propiedad de los autores)}

\section{DISCUSIÓN}

El diagnóstico de hernia de Amyand es muy difícil y usualmente se hace durante la intervención quirúrogica. Su presentación clínica se relaciona con un proceso herniario encarcelado (11-13).

De todas las hernioplastias del adulto, se halla el apéndice normal dentro del saco herniario en $1 \%$ y el apéndice inflamado, en $0,1 \%$. La mortalidad de esta condición varía entre 14 \% y $30 \%$, y se relaciona principalmente con las enfermedades asociadas $(6,7,14)$.

La presentación clínica depende del compromiso del saco herniario y del estado inflamatorio del apéndice. Se pueden encontrar, fiebre, emesis, distensión abdominal, dolor en la región periumbilical, que posteriormente se localiza en la fosa ilíaca derecha o en la región inguinal. También pueden presentarse síntomas de irritación peritoneal, en la mayoría de los casos localizados porque el orificio inguinal limita la extensión de la peritonitis $(5,15,16)$.

Con base en su experiencia, Losanoff y Basson propusieron una clasificación para facilitar el tratamiento (tabla 1) (5,17-20). Al respecto, se han planteado varias opiniones: algunos autores (21) proponen la apendicectomía profiláctica y afirman que disminuyen la morbilidad, la mortalidad y los costos, sin aumentar los riesgos quirúrgico y anestésico. Otros señalan que la resección de un órgano con contenido fecal viola la técnica aséptica con la posibilidad de aumento de las complicaciones infecciosas, en contraste con una cirugía limpia como la hernioplastia inguinal, cuyo tratamiento estándar es actualmente protésico $(8,22)$. 
Tabla 1. Clasificación Losanoff y Basson de la hernia de Amyand

\begin{tabular}{|c|c|c|}
\hline Clasificación (tipos) & Descripción & Tratamiento \\
\hline 1 & Apéndice normal en una hernia inguinal & Reducción de la hernia y colocación de malla \\
\hline 2 & $\begin{array}{l}\text { Apendicitis aguda en una hernia inguinal sin } \\
\text { sepsis abdominal }\end{array}$ & $\begin{array}{l}\text { Apendicectomía y reparación primaria de la } \\
\text { hernia sin malla }\end{array}$ \\
\hline 3 & $\begin{array}{l}\text { Apendicitis aguda en una hernia inguinal y sep- } \\
\text { sis de la pared abdominal }\end{array}$ & $\begin{array}{l}\text { Laparotomía, apendicectomía y reparación de } \\
\text { la hernia sin prótesis (malla) }\end{array}$ \\
\hline 4 & $\begin{array}{l}\text { Apendicitis aguda en una hernia inguinal con } \\
\text { enfermedad abdominal concomitante }\end{array}$ & $\begin{array}{l}\text { Igual que el tipo } 3 \text { más el tratamiento de la } \\
\text { enfermedad concomitante }\end{array}$ \\
\hline
\end{tabular}

\section{CONCLUSIÓN}

Por su infrecuencia, no hay protocolos estándar de tratamiento estándar para la hernia de Amyand. La inflamación del apéndice, el grado de contaminación, la edad y las comorbilidades del paciente y los hallazgos quirúroicos son determinantes para definir un adecuado tratamiento.

En nuestro paciente se hicieron apendicectomía y hernioplastia con aplicación de prótesis, sin complicaciones intra- ni postoperatorias. Esto se puede deber a que era un paciente joven, sin comorbilidades y con siognos inflamatorios iniciales en el apéndice cecal.

Es importante tener en cuenta la posibilidad de esta hernia, aunque sea infrecuente, e intervenir oportunamente al paciente; de lo contrario, el cuadro puede evolucionar hacia complicaciones como abscesos, fístulas, peritonitis, isquemia testicular, fascitis necrosante, necrosis del contenido herniario, obstrucción intestinal, choque séptico e, incluso, la muerte.

\section{CONFLICTOS DE INTERESES}

Ninguno por declarar

\section{REFERENCIAS BIBLIOGRÁFICAS}

1. Córdova A, Viscido G, Picón Molina H, Palencia R, Doniquian A. Hernia de Amyand: comunicación de dos casos reparados con técnica de Rutkow-Robbins. Rev Hisp Her. 2014 Jul-Sep;2(3):111-4. DOI 10.1016/j. rehah.2014.03.002.
2. Doyle GS, McCowan C. Amyand hernia: a case of an unusual inguinal herniace. Am J Emerg Med. 2008 Jun;26(5):637.e5-6. DOI 10.1016/j.ajem.2007.10.028.

3. Sancho Muriel J, Torregrosa Gallud A, García Pastor P, López Rubio M, Argüelles BG, Bueno Lledó JA. Hernia de Amyand: presentación de tres casos y revisión bibliográfica. Rev Hisp Her. 2016 Jul-Sep;4(3):107-11. DOI 10.1016/j.rehah.2015.05.001.

4. Valadéz-Caballero D, Enríquez Garibay JA, Ramírez Madera F, González Acosta J, Obregón Méndez J, Vega de Jesús M, et al. Hernia de Amyand en la era de la cirugía laparoscópica. Reporte de un caso y revisión de la literatura. Rev Mex Cir Endoscop. 2011 Jun;12(2):63-6.

5. Benavides de la Rosa DF, López de Cenarruzabeitia Í, Moreno Racionero F, Merino Peñacoba LM, Beltrán de Heredia J. Case report: Amyand's hernia, diagnosis to consider in a routine procedure. Rev Esp Enferm Dig. 2015 Nov;107(11):708-9. DOI 10.17235/ reed.2015.3816/2015.

6. Ramos Rodriguez J, Cruz Rodríguez J, Ramírez León C, O’Farril Hernández M. Hernia de Amyand. Presentación de un caso. Medisur. 2015 May;13(2):321-5.

7. Ivanschuk G, Cesmebasi A, Sorenson EP, Blaak C, Loukas M, Tubbs SR. Amyand's hernia: a review. Med Sci Monit. 2014 Jan;20:140-6. DOI 10.12659/ MSM.889873.

8. Smith-Singares E, Boachie JA, Iglesias IM. A rare case of apendicitis incarcerated in an inguinal hernia. J Surg Case Rep. 2016 Jun;2016(6). pii: rjw096. DOI 10.1093/jscr/rjw096.

9. Klobusicky P, Feyerherd P. Usage of a self-adhesive mesh in TAPP hernia repair: A prospective study based 
on Herniamed Register. J Minim Access Surg. 2016 Jul-Sep;12(3):226-34. DOI 10.4103/0972-9941.181388.

10. AI Maksoud AM, Ahmed AS. Left Amyand's hernia: An unexpected finding during inguinal hernia surgery. Int J Surg Case Rep. 2015;14:7-9. DOI 10.1016/j. ijscr.2015.06.029.

11. De la Peña-López J, Silgado-Kerouelen R, CalderónMorón H. Abdomen agudo por hernia de amyand. Rev Cienc Biomed. 2011 Mar;2(1):106-10.

12. Michalinos A, Moris D, Vernadakis S. Amyand's hernia: a review. Am J Surg. 2014 Jun;207(6):989-95. DOI 10.1016/j.amjsurg. 2013.07.043.

13. Oremule B, Ashrafi MH. Amyand's hernia with a periappendicular abscess. BMJ Case Rep. 2014 Apr 28;2014. pii: bcr2013203062. DOI 10.1136/bcr-2013203062.

14. Mora Quesada I, Barrantes León C. Caso I - 2015: Masculino de 63 años con hernia de Amyand. Rev Clín Esc Med UCR-HSJD. 2015 Nor;5(1):1-4.

15. Feitosa Cavalcante J, Melo Teixeira Batista H, Cavalcante Pita Neto I, Fernandes Frutuoso J, Rodrioues Pinheiro W, Maria Pinheiro Bezerra I, et al. Amyand's Hernia with Appendicitis: A Case Report and Integrative Review. Case Rep Surg. 2015;2015:941039. DOI 10.1155/2015/941039.

16. Sharma H, Gupta A, Shekhawat NS, Memon B, Memon MA. Amyand's hernia: a report of 18 consecutive patients over a 15-year period. Hernia. 2007 Feb; 11(1):31-5.

17. Ivanschuk G, Cesmebasi A, Sorenson EP, Blaak C, Loukas M, Tubbs SR. Amyand's hernia: a review. Med Sci Monit. 2014 Jan;20:140-6. DOI 10.12659/ MSM.889873.

18. Contreras Ruiz Velasco R, García La Rotta JA, González Avilés JM, Rojas Díaz O, Acevedo Estrađa RI, Murillo Zolezzi A. Hernia de Amyand del lado izquierdo: revisión de la bibliografía y comunicación de un caso. Rev Espec Méd-Quir. 2008 Abr-Jun;13(2):88-92.

19. Losanoff JE, Basson MD. Amyand hernia: a classification to improve management. Hernia. 2008 Jun;12(3):325-6. DOI 10.1007/s10029-008-0331-y.

20. García-Cano E, Martínez-Gasperin J, Rosales-Pelaez C, Hernández-Zamora V, Montiel-Jarquín JÁ, FrancoCravioto F. Hernia de Amyand y apendicitis complicada; presentación de un caso y elección de tratamiento quirúrogico. Cir Cir. 2016 Ene;84(1):54-7.

21. Morales-Cárdenas A, Ploneda-Valencia CF, SainzEscárrega VH, Hernández-Campos AC, NavarroMuñiz E, López-Lizarraga CR, et al. Amyand hernia: Case report and review of the literature. Ann Med Surg (Lond). 2015 Apr;4(2):113-5. DOI 10.1016/j. amsu.2015.03.007.

22. Singal R, Gupta S. "Amyand's Hernia" - Pathophysiology, Role of Investigations and Treatment. Maedica (Buchar). 2011 Oct;6(4):321-7. 